\title{
Notas sobre incertidumbre, flexibilidad, y ordenaciones de conjuntos
}

\author{
SALVADOR BARBERÀ \\ Departament d'Economia i d'Història Econòmica \\ Universitat Autònoma de Barcelona
}

\section{Introducción}

Muchas de nuestras decisiones nos cierran algunas puertas, dejando otras abiertas.

Aunque en ocasiones podamos prever con certeza los resultados de cada acción posible, en otras es más adecuado definir la naturaleza de nuestros actos como secuencias de decisiones que van alterando nuestros márgenes de actuación futura. Naturalmente, todo es cuestión del ámbito de decisiones que estemos considerando, y del horizonte a tener en cuenta. Según qué óptica adoptemos, elegir un destino para nuestras vacaciones puede verse como una decisión final, o como el comienzo de una etapa rica en sorpresas y elecciones futuras.

En esta nota quiero comentar distintos modelos de decisión que tienen algo en común: nuestras acciones determinan hoy qué oportunidades quedan definitivamente excluidas para el futuro, y cuâles serán aún posibles más adelante. Cada acción se caracteriza por su conjunto de alternativas no descartadas, y ordenar acciones se identifica, formalmente, con ordenar subconjuntos del conjunto universal de alternativas. Supondremos que los agentes tienen un orden de preferencias bien definido sobre el conjunto de alternativas, y estudiaremos distintas formas de extender dicho orden a su conjunto potencia.

Identificar decisiones con conjuntos de resultados no descartados es una característica común a varios modelos de decisión que divergen en otros aspectos. Nuestras acciones, hoy, determinan qué puede suce- der en el futuro; pero conviene entender de quién depende el resultado final, cómo van a seguir estrechándose en el futuro las opciones que hoy siguen abiertas. Aun entendiendo que nuestra elección del lugar de vacaciones deja abierto un abanico de opciones, no cs lo mismo pensar en el tiempo que vaya a hacer cuando efectivamente las disfrutemos, que en las excursiones entre las que vamos a poder escoger. En el primer caso las opciones a las que nos enfrentamos son inciertas, y será la meteorología quien determine el resultado final. En el segundo scguimos sicndo nosotros quienes, en el futuro, tendremos una nueva oportunidad de decidir. El propio ejemplo refleja la ambigüedad de la distinción, puesto que al decidir qué excursiones hacer también podemos tener en cuenta, eventualmente, el tiempo que haga: siempre habrá modelos más generales que engloben a los que ya conocemos. Pero aquí básicamente nos ocuparemos de los dos casos apuntados: aquel en que, una vez tomada nuestra decisión actual, la opción final queda en manos de la naturaleza, y aquel en que, más adelante, podremos influir de nuevo sobre el curso de los acontecimientos.

Mi intención al presentar someramente el material que sigue es doble. Por una parte, dar noticia de una literatura reducida pero interesante, desarrollada principalmente entre economistas. Por otra, incluir los modelos que presento dentro de una clase más amplia de ejercicios, caracterizados todos ellos porque se interesan en la extensión de órdenes sobre un conjunto al conjunto potencia. 


\section{Algunos ejemplos, y un marco general}

Supongamos que prefiero $\mathrm{x}$ sobre z. ¿Cómo valorar el conjunto $\{\mathrm{xz}\}$ ?

Una posibilidad es declarar que $\{x\} y$ $\{x z\}$ son indiferentes entre sí, y preferidos a $\{z\}$. Esta posibilidad es consistente con la siguiente interpretación: $x$ y $z$ son dos opciones mutuamente excluyentes, dos alternativas entre las que voy a elegir. Como prefiero $\mathrm{x}$ sobre $\mathrm{z}$, voy a elegir $\mathrm{x}$ tanto si puedo optar entre ambas (c interpreto el conjunto $\{x z\}$ como esta posibilidad), como si mi única opción es $\mathrm{x}$ (representada por el conjunto $\{x\}$ ).

Otra posibilidad es declarar que el conjunto $\{x z\}$ es superior a $\{z\}$, y también a $\{x\}$. Podríamos justificarla de varias formas. Aún interpretando que $\{x z\}$ representa la opción de escoger entre $x$ y $z$, podríamos entender que la elección definitiva entre estas dos opciones deba producirse en un futuro incierto, y que, ante esta incertidumbre, es preferible tener abiertas las posibilidades representadas por $\{x z\}$ que la única opción representada por $\{x\}$. Las fuentes de aquella incertidumbre pueden ser varias: mis preferencias pueden cambiar, y en tal caso es bueno poder tener la opción $z$ aún abierta, además de la $x .0$, incluso si mis preferencias se mantienen, es posible que $x$ deje de estar disponible, $y$ que $z$ aparezca como una buena solución de recambio.

Entender que $\{x z\}$ es superior a $\{z\}$, $y$ también a $\{x\}$, puede tener otras justificaciones, de raíz distinta. Las anteriores suponian que $x \mathrm{y} z$ son opciones excluyentes entre las que se debe elegir. Pero podríamos considerar que estos dos elementos son mutuamente compatibles: por ejemplo, dos candidatos a formar parte de una sociedad honorifica. En tal caso, si tanto $\mathrm{x}$ como $\mathrm{z}$ son candidatos merecedores de la distinción, y el conjunto $\{x z\}$ simboliza la elección de ambos, $\{x z\}$ será mejor que $\{x\}, y$ también mejor que $\{z\}$.
La anterior interpretación nos lleva, naturalmente, a poder justificar otros 6 rdenes, por ejemplo aquel en que $\{x z\}$ sea peor que $\{z\}, y$ por tanto también peor que $\{x\}$. Basta con pensar de nuevo en la misma elección, pero entendiendo ahora que ni $x$ ni $z$ son merecedores del honor, aunque su concesión a $x$ sea menos injusta que si z lo recibe: en cualquier caso, lo peor puede ser que ambos lo obtengan. O no. También podría ser que, dados los pocos méritos de $\mathrm{z}$, la elección simultánea de ambos candidatos $\{\mathrm{xz}\}$, sea un compromiso mas aceptable que la elección de $z$ solamente, y a la vez menos deseable que la de $x$ en solitario. En tal caso, $\{x z\}$ se encontraría entre $\{x\}$ y $\{z\}$.

Una justificación distinta de este mismo orden, en que $\{x z\}$ ocupa un lugar intermedio entre $\{x\}$ y $\{z\}$, sc obtiene naturalmente si, de nuevo, consideramos que $x$ y $z$ son mutuamente excluyentes e interpretamos que $\{x z\}$ es el conjunto de posibles resultados de una lotería con probabilidades desconocidas, mientras que los conjuntos con un único elemento garantizan que éste sea elegido. En tal caso, si preferimos a $x$ sobre $z$, es natural que prefiramos la certeza de $\mathrm{x}$ a cualquier lotería, mientras que la certeza de $z$ debería ser menos atractiva que el par $\{x z\}$.

Finalmente, y aunque interpretemos que la naturaleza escogerá entre las alternativas posibles con probabilidades desconocidas, un agente extremadamente pesimista podría entender que, en todo caso, obtendrá aquella que le sea más desfavorable. En tal caso, $\{x z\}$ sería indiferente a $\{z\}$ y peor que $\{x\}$.

Vemos, pues, que la sencilla pregunta con que iniciamos esta sección admite múltiples respuestas. Con este breve repaso de posibilidades, he pretendido llamar la atención sobre situaciones que tienen elementos formales en común: una ordenación sobre un conjunto $\mathrm{A}$ de elementos $(x, z, \ldots)$ debe extenderse al conjunto potencia de $\mathrm{A}$, permitiendo así comparaciones 
entre subconjuntos. He querido destacar también que el problema formal de cxtender un orden sobre un conjunto a su conjunto potencia admite interpretaciones muy diversas: importa qué representa la relación entre elementos, cómo interpretamos los conjuntos, qué tipo de problema deseamos modelar y para qué. Ya hemos visto casos en los que las alternativas son mutuamente excluyentes, y otros en que no lo son. Casos en los que interviene el azar, $y$ otros deterministas, etc. $\mathrm{Y}$ aún podríamos añadir otros en los que la relación binaria de partida no debe entenderse como la preferencia de nadie: por cjemplo, $\{x\}$ y $\{z\}$ pueden ser conjuntos de proposiciones, $\{x z\}$ la unión de aquellos, y la relación binaria entre conjuntos de proposiciones representar la plausibilidad dc cada combinación!.

En las páginas siguientes expondré con más detalle algunos resultados sobre la extensión de órdenes desde un conjunto a su conjunto potencia, ligados a interpretaciones concretas de nuestro marco general de referencia. El objetivo es explorar el rendimiento de un formalismo sencillo, que permite abordar muchos temas distintos con un mismo lenguaje, y a la vez advertir contra su uso indiscriminado, insistiendo en la importancia de acompañarlo, en cada caso, con interpretaciones concretas que permitan distinguir entre axiomas o propiedades alternativas.

Terminaré, pues, proponiendo un marco general, que se irá haciendo más específico en los apartados siguientes.

Sea $\mathrm{K}$ un conjunto finito de objetos. Los símbolos $\mathrm{X}, \mathrm{z}, \mathrm{w}, \ldots$ representan objetos en K.

Sea nes sobre K. Llamamos órdenes a las relaciones binarias completas, reflexivas y antisimétricas. Los símbolos $\mathrm{P}, \mathbf{P}^{\prime}$... representan órdenes sobre $\mathrm{K}$.

Denotamos por $2^{\mathrm{K}}$ el conjunto potencia de $K$, es decir, el formado por todos los subconjuntos de K. En ciertas aplicaciones donde carece de interpretación interesa excluir cxplícitamente cl conjunto vacío, que forma parte de $2^{\mathrm{K}}$ si no indicamos lo contrario. Llamamos conjuntos unitarios a aquellos que contienen exactamente un elemento de K. Dado un conjunto $\mathrm{C} 2^{\mathrm{K}}$, $y$ un orden $P$ sobre $K$, denotaremos por $\max (\mathrm{A})$ y por $\min (\mathrm{A})$, respectivamente, el elemento maximal y el minimal de $\mathrm{C}$ respecto a $P$.

Sea 稆 el conjunto de las relaciones binarias reflexivas sobre $2^{\mathrm{K}}$, definidas sobre al menos todos los subconjuntos unitarios. Los símbolos $\geq, \geq^{3}, \ldots$ representan relaciones en $\mathfrak{X}$. Cada relación $\geq$ induce otras dos:

$(\succ)$, definida de modo que $\mathrm{VC}$, $C^{\prime} \quad 2^{\mathrm{K}},\left(\mathrm{C}>\mathrm{C}^{\prime}\right) \leftrightarrow\left(\mathrm{C} \geq \mathrm{C}^{\prime} \& \mathrm{C}^{\prime} \neq \mathrm{C}\right)$

$(\sim)$, definida de modo que $\mathrm{rC}$, $\mathrm{C}^{\prime} \quad 2^{\mathrm{K}}, \mathrm{C} \sim \mathrm{C}^{\prime} \longrightarrow\left(\mathrm{C} \geq \mathrm{C}^{\prime} \& \mathrm{C}^{\prime} \geq \mathrm{C}\right)$

Una relación $\geqslant$ es casi transitiva si su relación inducida $\succ$ es transitiva.

Diremos que $\geqslant$ es una preorden si es reflexiva, transitiva y completa.

Una relación 38 sobre $2^{\mathrm{K}}$ es una extensión de $P$, definida sobre $K$, si para todo par de objetos $x, z, x$ Py implica $x \succ y$.

\section{Los conjuntos como expresión \\ de incertidumbre sobre resultados}

El primer contexto que queremos analizar es aquel en que, una vez tomada una acción, ciertas altemativas quedan excluidas, y el resultado final entre las aún posibles se determina aleatoriamente, sin participación del agente decisor. Esta descripción, para ser precisa, debe indicar quế sabe el decisor acerca del mecanismo aleatorio que vaya a ser utilizado. Lo clásico es suponer que los agentes conocen qué consecuencias tienen sus acciones en cada estado de la naturaleza, y son capaces de atribuir probabilidades, subjetivas u objetivas, a cada estado; pero aquí vamos a modelizar situaciones en que el decisor sea totalmente ignorante respecto a aquel 
mecanismo. Esta situación cxtrema formalizaría el concepto de incertidumbre, frente al más habitual de ricsgo, caracterizado precisamente por la disponibilidad de información adicional sobre las probabilidades.

Identificaremos cada conjunto de alternativas con una acción, entendiendo que una vez tomada ésta las alternativas del conjunto aún son posibles, y las demás ya han quedado excluidas. Entenderemos que, una vez adoptada la acción y acotado el conjunto de alternativas aún posibles, una de ellas será elegida aleatoriamente, según un mecanismo del que el decisor ni sabe ni cree saber nada. Si conocemos las preferencias del decisor sobre las alternativas, ¿qué preferencias sobre acciones, es decir, sobre conjuntos de alternativas, es plausible atribuirle?

Una posible respuesta consiste en proponer algún criterio concreto para formar relaciones de preferencia sobre conjuntos partiendo de una ordenación de alternativas. Distintas versiones de los criterios maximin, leximin o maximax, que comentaremos más adelante, podrian avanzarse como propuestas «razonables». Pero cada propuesta debería venir acompañada de razones que la avalasen; ya sea de una descripción del proceso implícitamente seguido por los decisores para evaluar distintos conjuntos, ya sea de una lista de propiedades que satisfaga el criterio adclantado. Por ello parece interesante empezar al revés: proponiendo axiomas que parezca razonable imponer a las preferencias de los decisores sobre conjuntos, en relación a su ordenación de alternativas. Desde luego, y dado que interpretamos cada conjunto singular como la certeza de que su elemento resulte elegido, requeriremos que las preferencias del decisor sobre conjuntos sean una extensión de sus preferencias sobre alternativas a todos sus subconjuntos no vacíos.

A este primer requisito podríamos añadirle olros. El siguiente axioma fue propuesto por Gardenförs ${ }^{2}$ :
(GF) Sea $A$ un subconjunto de $K$, y $x \in K \backslash A$. Si $x P \max (A)$, entonces $A \cup\{x\}>A$, y si $\min (A) P x$, entonces $A \succ A \cup\{x\}$.

Scgún el principio de Gardenförs, si a un conjunto de posibles resultados le añadimos otro que es mejor (resp. peor) que todos cllos, el conjunto ampliado será mejor(resp. pcor) que el anterior.

Otro requisito aparentemente plausible queda reflejado en el siguicnte axioma de monotonia:

(M) Si $A, B, C \in 2^{K} \phi, A \cap B=A \cap C=$ $=\phi, y B \succ C$, entonces $A \cup B \succ A \cup C$.

Este principio refleja cierto grado de separabilidad: con calificaciones, expresa que añadir un buen conjunto no puede ser peor que añadir otro menos atractivo.

Acaso el lector ya se haya inquietado ante estos axiomas. ¿Por qué suponerlos? ¿Reflejan bien las valoraciones que haria de cada conjunto un decisor sometido a incertidumbre? Posiblemente este fuera también el sentir de Kannai y Peleg, quienes concretaron su incomodidad demostrando la siguiente ${ }^{3}$

Proposición 1 (Kannai y Peleg). Si K contiene al menos cinco alternativas, no existe ningún preorden $\geqslant$ sobre $2^{\mathrm{K}} \phi$ que satisfaga los axiomas (GF) y (M).

Algo nos dice este resultado. Curando se trata de interpretar conjuntos de alternativas como listas de consecuencias posibles en situaciones de incertidumbre, nuestra intuición sobre cómo evaluar posibles combinaciones de situaciones inciertas puede traicionarnos fácilmente. Antes de precipitarnos en esta dirección, podríamos buscar axiomas parecidos, que siguieran reflejando, con posibles modificaciones técnicas, intuiciones análogas. Los siguientes axiomas se proponen con este espíritu.

Un primer axioma relaja los requisitos del principio de Gardenförs al caso en que sólo se comparan conjuntos de baja dimensiôn: 
(B) Para todo par de alternativas distintas $x, y \in K, x P y$ implica que $\{x\} \succ\{x, y\}$ $y$ que $\{x, y\} \succ\{y\}$.

El segundo axioma es intermedio entre (GP) y (B):

(K) Sean $A, B \in 2^{K}$. Si, para todo $x \in A$, $y$ para todo $y \in B$ se cumple $x P y$, entonces $A>B$.

Los axiomas $(B)$ y $(\mathrm{K})$ fueron introducidos por Barberà y por Kelly, respectivamente, para el estudio de correspondencias de elección social no manipulables ${ }^{4}$.

Consideremos también una versión algo más fuerte del axioma de monotonía, que no parece ni más ni menos plausible que el anterior:

(M) Si $A, B, C \in 2^{K}, A \cap B=A \cap C=\phi$, y $B \succ C$, entonces $A \cup B \succ A \cup C$.

Las dos proposiciones siguientes demuestran lo tenue que es la frontera entre posibilidad $\mathrm{e}$ imposibilidad en este terreno ${ }^{5}$.

Proposición 2. Existe un preorden sobre $2^{\mathrm{K}}$ 中 que satisface (K) y (M).

Proposición 3. Si K contiene al menos tres elementos, ninguna relación binaria sobre ${ }^{\mathrm{K}}$ $\phi$ puede satisfacer los axiomas (B) y (M').

Si nos parece suficiente $(K)$ en lugar de (GF), queda margen para explorar qué extensiones de $\mathrm{R}$ al conjunto potencia de $K$ pueden reflejar actitudes hacia la incertidumbre que respeten los axiomas de la proposición 2. Pero todo es muy frágil. La proposición 3 es contundente: si se intenta reforzar el requisito de monotonía con el paso, aparentemente anodino, de (M) a (M'), dejan de existir relaciones binarias, incluso entre aquellas que no sean ni preórdenes, capaces de satisfacer ni tan sólo la condición (B), la más débil de las consideradas en su clase.

La ventaja de los resultados formales cs que ahí están, y cada cual puede interpretarlos a su manera. Mi conclusión, en vista de los anteriores, es que si queremos reflejar la incertidumbre de los agentes mediante sus preferencias sobre conjuntos de consecuencias no excluidas, debemos scr muy cuidadosos y explicitar en qué argumentos basamos nuestros axiomas. Estamos utilizando un modelo muy pobre, y resulta fácil caer en el error de exigir el cumplimiento simultánco de axiomas que se justifican cada uno por separado bajo interpretaciones distintas, pero que no tienen por qué ser coherentes entre sí.

Una posible historia que acompañe nuestro intento de ordenar conjuntos de alternativas es la siguiente. Supongamos que, además de ordenar sucesos ciertos, los agentes están efectivamente dotados de preferencias sobre loterias, que satisfacen los axiomas de Von Neumann y Morgenstern, y que valorarían cada conjunto por su utilidad esperada, si conocieran las probabilidades de elección de cada alternativa Pero consideran que cualquier lotería sobre los resultados es plausible, y no quieren tampoco atribuir probabilidades a dichas loterías. iEstamos dispuestos a atribuirles preferencias sobre conjuntos, basándonos en esta interpretación? Algunos de los axiomas propuestos, como (B) y (K), son compatibles con suponer que los decisores se rigen por cualquiera de las preferencias Von Neumann-Morgenstern compatibles con sus preferencias ordinales, y que mantendrían la misma valoración relativa cualquiera que fuese la lotería que acabase determinando los resultados. Así, por ejemplo, cualquier maximizador de utilidad esperada que prefiera a $\mathrm{x}$ sobre y considerará que darle alguna probabilidad de elección a y es mejor que no dársela, y que la posibilidad de que $x$ sea escogida es mejor que la certeza de $y$, si éstos son los dos únicos resultados en juego. Esta sería la justificación de (B), y la de (K) es análoga. Esta interpretación permite comparar entre sí algunos conjuntos de alternativas, pero no todos. En muchos casos la comparación entre las uti- 
lidades esperadas de dos conjuntos de resultados dependerán crucialmente de las actitudes de los agentes ante el riesgo, y de probabilidades que suponemos desconocidas. Volvamos sobre el axioma (GF): si añadir una alternativa mejor a todas altcrase las probabilidades de elección de las anteriormente consideradas -por ejemplo, aumentando mucho la probabilidad de la peor entre ellas-, no está claro que aquella adición fuese deseable. De modo similar, las condiciones de monotonía sólo se satisfarian para cualquier maximizador de utilidad esperada si éste pudiese tener garantía de que ciertas proporciones entre las probabilidades de las alternativas originales no se viesen alteradas por la presencia de otras nuevas. Así pues, sólo algunas comparaciones de conjuntos resultan inequívocas para un maximizador de utilidad esperada. Esto sugiere varias posibles líneas de desarrollo.

En primer lugar, podríamos admitir que, en un marco tan esquemático como el disponible, donde sólo se distingue entre aquellas alternativas definitivamente excluidas y las que aún podrían materializarse, resulta excesivo requerir una extensión completa de las preferencias sobre un conjunto a todos sus subconjuntos no vacíos. Podríamos limitarnos a caracterizar aquellas comparaciones que resultan inequívocas bajo determinadas «historias» sobre el posible razonamiento de un decisor bajo condiciones de incertidumbre. Poco más allá de (K) podremos ir si exigimos que toda comparación sea coherente con cualquier escala de utilidad y cualquier lotería arbitraria. Pero, partiendo de axiomas que requieren diferentes grados de completitud para satisfacerse, podriamos buscar distintas interpretaciones que las justificasen. Si seguimos con la historia anterior, podemos preguntarnos bajo qué familias de distribuciones es cierto que todas las comparaciones exigidas para que el axioma (GF) se satisfaga bajo cualquier escala de utilidades son inequívocas. Esta línea de trabajo es análoga a la seguida por diversos autores para contextos algo más estructurados, al suponer que la incertidumbre de los agentes es parcial, y se refleja en que las probabilidades de ocurrencia de cada alternativa se mueven dentro de ciertos intervalos ${ }^{6}$.

Para aquellos que piensen que renunciar a extensiones completas de las preferencias, $y$, por tanto, dejar ciertos conjuntos sin comparar, es eludir la cuestión, y declararse incapaz de modelar adecuadamente el comportamiento bajo incertidumbre, queda abierta la posibilidad de no exigir que las preferencias sobre conjuntos sean $\tan$ coherentes como las comparaciones de alternativas en que se basan. Aunque partamos de órdenes sobre alternativas, ya hemos admitido que las preferencias sobre conjuntos puedan ser preórdenes. ¿Por qué no relajar algo más esta exigencia, ya que en nuestro universo finito basta con que una relación sea acíclica para dar lugar a elecciones no vacías? Las tensiones entre axiomas del mismo tipo que los anteriomente considerados pueden rebajarse si exigimos que nuestra extensión sólo sea un cuasi-orden. Existen cuasi-órdenes sobre conjuntos que extienden órdenes sobre alternativas satisfaciendo (GF) y (M), algunos de los cuales han sido axiomatizados por Bossert?

En los párrafos anteriores hemos comentado una posible fuente de interpretaciones para nuestros órdenes de conjuntos, basadas en suponer que los individuos, en situaciones de ignorancia, emiten juicios coherentes con aquellos que les guiarían, de ser maximizadores de utilidad esperada, si conocieran aquellas probabilidades que ignoran. Pero no hay por qué relacionar tan estrechamente los enfoques de riesgo y de incertidumbre. Podemos profundizar en las consecuencias de suponer que, al comparar conjuntos de resultados inciertos, los agentes respetan criterios sencillos, como los expuestos en nuestros axiomas. Asi, por ejemplo, hemos 
visto que la Proposición 2 deja abierta la puerta a extensiones que satisfagan $(\mathrm{K})$ y (M). ¿En qué tipos de reglas se basan? La siguiente proposición nos indica que, incluso relajando (K) a (B), el margen es estrecho: debemos comparar conjuntos atendiendo a sus elementos maximales y minimales, tan sólo.

Proposición 4. Sea un preorden $\geq$ sobre $\left.2^{\mathrm{K}}\right\rangle_{\phi}$, que extienda a un orden $\mathrm{P}$ respetando (B) y (GF). Para todo subconjunto $A$, tendremos que $A-\{\max (A), \min (A)\}$.

Esta proposición demuestra la fuerza de los axiomas: sólo admiten reglas que declaren indiferentes a los conjuntos con un mismo máximo y un mismo mínimo. Esta conclusión reproduce, en un marco mucho más pobre, la que ya habian alcanzado Arrow y Hurwicz ${ }^{8}$ en el contexto estándar de teoría de la decisión. Puede lecrse de modo muy positivo: justifica, de modo muy sencillo, el uso de criterios clásicos como el maximin o el maximax, propuestos mucho antes, sin necesidad de recurrir a consideraciones explícitas de extrema aversion o preferencia hacia el riesgo. En Barberà, Barrett y Pattanaik ${ }^{9}$ se caracterizan todas las reglas que satisfacen aquellos axiomas. Bossert ${ }^{10}$ propone clasificaciones entre reglas de extensión en términos de aversión a la incertidumbre, en paralelo a las clásicas medidas de aversión al riesgo.

Finalmente, otros autores se desmarcan de las anteriores combinaciones de axiomas, y caracterizan otras reglas de extensión "razonables», dejando abierta para el lector si éste considera o no adecuada cada una de ellas como criterio para resolver problemas en condiciones de incertidumbre, o para describir el comportamiento de algunos agentes en tales circunstancias. Dos ejemplos interesantes vienen dados por la extensión lexicográfica del criterio maximin (Pattanaik y Peleg) "l y la extensión basada en el elemento mediano de cada conjunto respecto al orden de preferencias (Nitzan y Pattanaik) ${ }^{12}$. Cada una de estas reglas queda únicamente descrita por los axiomas que satisface, dejando al descubierto las limitaciones y el alcance de diversas teorías del comportamicnto bajo condiciones de incertidumbre que se basen en un punto de partida tan sencillo como resumir todo cuanto conocemos sobre nuestras acciones a través del conjunto de consecuencias no descartadas.

\section{Los conjuntos como expresión de incertidumbre sobre las oportunidades de elección en el futuro}

Si hoy tomamos una decisión, ciertas oportunidades futuras quedan excluidas, pero no otras. Siguiendo con nuestro ejemplo de vacaciones, si decidimos dirigirnos a Italia en automóvil, dejamos atrás la oportunidad de viajar a París o a Londres, pero aún se nos abren múltiples posibilidades. Muchas decisiones tienen este aspecto, al menos dentro de horizontes temporales intermedios: suficientemente largos para que quepa aún concretar mejor nuestras acciones, y lo bastante cortos para que no podamos rectificar las consecuencias de lo que ya hicimos. De nuevo, esta observación nos lleva a considerar conjuntos de alternativas no excluidas. Pero vamos a suponer que el decisor sigue teniendo control sobre los resultados abiertos, que va a tener oportunidad de elegir de nuevo entre aquello que no ha descartado aún: al pasar la frontera italiana no será la naturaleza, sino el mismo, quien decida si ir hacia el Este o hacia el Sur.

Formalmente, nos planteamos la cuestión de extender órdenes sobre un conjunto al conjunto potencia, de forma coherente con la interpretación apuntada: cada conjunto viene asociado a una acción presente, $\mathrm{e}$ indica aquellas altemativas sobre las que aún podremos escoger en el futuro. Supondremos, además, que tiene sentido hablar del conjunto vacío como falta total de oportunidades, y que éste es peor que cualquier otro. 
Si nuestras preferencias no van a variar, de modo que nuestra elección futura será aquella misma que hoy hariamos si hubiera que seleccionar una sola alternativa, parcce que la ordenación de conjuntos deba seguir una pauta clara: identificar cada uno de ellos con la mejor de las alternativas que contiene, y declarar a un conjunto mejor que otro siempre y cuando el maximal de uno sea mejor que el otro; por ello mismo, si dos conjuntos tienen el misno elemento maximal, deberían considerarse indiferentes.

A pesar de lo anterior, la idea de que conjuntos más amplios son mejores que otros más reducidos, incluso si ambos tienen un mismo maximal, resulta atractiva por diversas razones, algunas de las cuales vamos a exponer.

Ante todo, mencionemos un conjunto de justificaciones en las que no nos detendremos demasiado, y según las cuales un conjunto de elección más amplio ofrece mayor libertad de elección, y es por ello mismo preferible a otro más reducido, incluso si nuestra clección bajo uno y otro acaba siendo la misma. Diversos autores ${ }^{13}$, han insistido en el valor intrínseco de las posibilidades de elección abiertas ante nosotros, más allá del que tengan como paso intermedio hacia decisiones más o menos favorables. Algunas axiomatizaciones de la idea de libertad de elección como valor intrínseco a conjuntos de alternativas pueden conducir fácilmente a resultados triviales. Pattanaik y $X u^{14}$ lo demuestran magistralmente, al probar que si consideramos que cualquier conjunto singular ofrece igual libertad de elección que los demás (es decir, ninguna, ya que contienen un solo elemento), y que cualquier superconjunto ofrece mayor libertad que los conjuntos que contiene, basta con un axioma técnico adicional para que la libertad de elección quede identificada con la cardinalidad de los conjuntos. Decir que cualquier conjunto con $\mathrm{n}$ elementos ofrece mayor libertad que cualquiera con $n-1$, y menos que los conjuntos con $n+1$, no supone demasiada sutileza. $Y$, sin embargo, resulta de axiomas que parecen incontrovertibles. Otros muchos autores han intentado refinar el sentido preciso que podría darse al valor de los conjuntos de elección, como algo que trasciende a las propias elecciones. Nosotros vamos a dar noticia, sin embargo, de aquellos enfoques que podrían justificar una valoración superior de los conjuntos mayores atendiendo solamente a consideraciones de utilidad.

Concretamente, dado un preorden sobre $2^{\mathrm{K}}$, diremos que satisface el axioma de inclusión si es cierto que ordena a cualquier conjunto como estrictamente mejor que sus subconjuntos propios.

¿Bajo qué interpretaciones podemos explicar preórdenes de conjuntos que satisfagan el axioma de inclusión? Una explicación muy extendida se basa en la idea de flexibilidad. Tener más donde elegir puede ser interesante, sobre todo si no estamos del todo seguros de nuestras prefercncias o necesidades futuras. Muchos argumentos conservacionistas se basan en la posibilidad de que el futuro nos descubra nuevos usos de las especies que hoy desconocemos pero podríamos estar destruyendo irreversiblemente si adoptásemos determinadas políticas agresivas para nuestro entorno. $\mathrm{O}$, volviendo a nuestro ejemplo más trivial de las vacaciones, factores internos (nuestro estado de ánimo) o externos (el estado del tiempo) pueden influir sobre nuestras preferencias en el momerito de la elección, y siempre será mejor poder contar con más opciones que con menos. Estas ideas, que cuentan con una larga tradición, fucron objeto de tratamiento axiomático por David Kreps ${ }^{15}$. Claramente, si un decisor no conoce sus preferencias futuras, pero tiene una distribución de probabilidad sobre cuáles vayan a ser sus preferencias entre las que satisfacen los axiomas de Von Neumann y Morgenstern, puede calcular el valor cspcrado de sus mejores elecciones entre 
los elementos de un conjunto, y compararlo con el de sus mejores elecciones bajo un conjunto alternativo. Esto genera, para cada lotería sobre las preferencias posibles de un decisor, un preorden sobre conjuntos de alternativas. Dicho preorden satisfará, sin duda, el axioma de inclusión, ya que una misma lotería dará lugar a elecciones iguales o mejores sobre un conjunto que sobre cualquiera de sus subconjuntos. Lo que Kreps demuestra es que la observación inversa también es cierta: para cualquier preorden dado sobre conjuntos de alternativas, y que satisfaga el axioma de inclusión, es posible encontrar una distribución sobre preferencias que dé lugar a aquel preorden mediante el tipo de construcción que acabamos de describir. En otros términos: el axioma de inclusión es la única restricción impuesta a los preórdenes sobre conjuntos si las preferencias por la flexibilidad se basan en la incertidumbre acerca de nuestras preferencias futuras ${ }^{16}$.

Al exponer las razones de Kreps para justificar que un conjunto sea mejor que sus subconjuntos hemos abandonado nuestra referencia a un orden sobre las alternativas, pasando a considerar la posibilidad de que nuestras acciones futuras no se guien por un criterio ya establecido. Con ello nos hemos alejado de nuestro marco de referencia: la extensión de preferencias sobre alternativas a conjuntos de alternativas. iEs posible encontrar, dentro de nuestro marco, razones para que, si bien las preferencias están fijadas, los agentes prefieran conjuntos mayores a otros más reducidos?

En una investigación conjunta con Birgit Grodal ${ }^{17}$, aún no concluida, he investigado esta cuestión, siguiendo una nueva línea interpretativa. Nuestra incertidumbre futura puede referirse a contingencias que hagan desaparecer algunas de las oportunidades de elección que nuestras propias acciones no habian descartado. Por ello, aunque estemos seguros de cuáles vayan a ser nuestras opiniones mañana, podemos preferir tener abierto un menú de posibilidades más amplio que otro más estrecho. Si algunas de las opciones no descartadas acaba siendo inviable, no nos perjudicará tener un conjunto más amplio sobre el que elcgir.

Para formalizar esta idea, que llamaremos preferencia por mayores oportunidades, consideraremos que, provisto de sus preferencias sobre alternativas, cada agente valora un conjunto $A$ del siguiente modo. Valora la probabilidad de que cada uno de los subconjuntos B de A acabe siendo el de las oportunidades sobre las que escoger (o, de modo equivalente, la probabilidad de que los elementos de A-B dejen de estar disponibles). Calcula su mcjor opción sobre cada subconjunto $\mathrm{B}$, y atribuye al conjunto A el valor esperado de aquellas decisiones óptimas, dadas las probabilidades de tener que escoger dentro de cada uno de sus subconjuntos. Claramente, dada una distribución de probabilidades de sobrevivencia para cada subconjunto de cada conjunto $\mathrm{A}$, y una función de utilidad VN-M que represente las preferencias de nuestro decisor, éste podră clasificar a todos los conjuntos de alternativas en términos de utilidad esperada, y el preorden resultante satisfará el axioma de inclusión. ¿Impone esta interpretación alguna propiedad adicional sobre los preórdenes de alternativas compatibles con ella? No, a menos que queramos satisfacer restricciones adicionales. De hecho, una simple reinterpretación de los resultados de Kreps nos permite concluir que, para cada preorden sobre conjuntos que satisfaga la propiedad de inclusión, puede encontrarse una distribución de probabilidad sobre los subconjuntos de cada conjunto para la cual el preorden inicial se obtiene como resultado de las operaciones antes descritas.

¿Qué ganamos, pues, interpretando las preferencias sobre conjuntos como resultado de preferencias por mayores oportunidades? Ante todo, observar que la 
incertidumbre futura no tiene por qué referirse a las preferencias, y que puede afectar a la disponibilidad de ciertas opciones: bien está que reconozcamos que nuestras acciones llevan a descartar ciertas oportunidades, pero también es adecuado tener en cuenta que otras podrían dejar de estar disponibles por otras razones. Por otra parte, esta interpretación encaja más de cerca con el modelo inicial, que relaciona preferencias sobre alternativas con preferencias sobre conjuntos. $Y$, finalmente, abre paso a otras preguntas, a las que de momento no tenemos contestación precisa. Por ejemplo: $\delta$ qué restricciones adicionales sobrevienen a los preórdenes sobre conjuntos si la mortalidad de cada alternativa es independiente de la de las demás? Sabemos que este requisito, a veces natural, sobre las posibles distribuciones, limita los preórdenes posibles, pero aún no disponemos de una caracterización completa.

\section{Comentarios finales}

Este breve repaso nos ha llevado a apuntar la existencia de un marco formal que, aunque muy pobre, contiene suficientes elementos para modelar algunos elementos básicos para la toma de dccisiones: la exclusión en el futuro de ciertas opciones, como resultado de nuestras acciones presentes, el papel del azar y de los decisores al determinar qué alternativas perviven, $y$ cuáles son escogidas en períodos futuros, etc. $Y$ en este marco se han sugerido inter- pretaciones distintas de lo que puede significar la incertidumbre como un tipo de ignorancia cualitativamente distinta que el riesgo. Hemos insinuado también que este mismo marco formal -el estudio de las extensiones de preferencias desde un conjunto a su conjunto potencia- puede servir para discutir otros temas de interés, citando al menos dos de ellos: el valor intrínseco de la libertad de elección y la plausibilidad de conjuntos de proposiciones, en su relación con la plausibilidad de cada una de cllas.

La fuerza del enfoque, y su debilidad, radican en lo mismo. Es destacable que estructuras tan elementales permitan modelar fenómenos interesantes y variados, y sorprenden las analogías entre problemas que en principio no tienen por qué estar relacionados. Esta misma versatilidad para describir problemas diversos encierra sus peligros. Es conveniente conservar en cada caso una interpretación concreta como punto de referencia para cualquier cjercicio formal, sobre todo en casos como éste, donde un mismo modelo puede encubrir historias tan distintas que los axiomas naturales para uno no tengan sentido para otro.

Aun cuando el estudio de las decisiones bajo condiciones de incertidumbre ha florecido en direcciones diversas y mäs sofisticadas que las aqui apuntadas, algunas de las ideas más básicas pueden expresarse en el lenguaje sencillo de las relaciones binarias sobre conjuntos. Es cuanto quería ilustrar en esta nota. 


\section{NOTAS}

1 Packard, D. (1981): «Plausibility Orderings and Social Choice», Synthese, núm, 49, pp. 415-418. También van en esta direceión muchos de los trabajos recientes de J, C. García Bermejo.

2 Gardenförs, P. (1976): wanipulability of Social Choice Functions», Sournal of Economic Theory, núm. 13, pp. 217-218.

* Y. Kannai y B. Peleg (1981): "A Note on the Extension of an Order on a Set to the Power Setw, Joumat of Economic Theon:

4 S. Barberà (1977): "The Manipulation of Social Choice Mechanisms that do not Leave "too much" to Chance», Econometrica, núm, 45, PP. 1573-1588.

J. Kelly, (1977): aStrategy-Proofness and Social Choice Functions without Single-valuedness", Econometrica, núm. 45, pp. 439.446 .

s Estos resultados están extraidos de Barberà y $\mathbf{P}$. Pattanaik (1984): «Extending an Order on a Set to the Power Set: some Remarks on Kannai and Peleg's Approach», Joumal of Economic Theory, núm. 32, pp. 185-191. El mismo número del Joumal of Economic. Theory contiene, junto al artîculo de Kannai y Peleg ya citado, diversos comentarios por parte de otros autores.

- Estamos pensando en trabajos como T. F. Bewley (1986): Knightion Decision Theay, Part I, Cowles Foundation DP 807; K. Nehring (1992); woundation for the Theory of Rational Choice with Vague Priors", en J. Geweke (ed.): Decision Making under Risk and Uncenainty, Dordrecht: Kluwer; D. Schmeidler (1989); «Subjective Probability and Expected Utility without Additivity", Econometrica, búm. 57, pp. 571-587. P. Walley (1991): Statistical Reasoning with Imprecise Probabilities, London: Chapman and Hall.

${ }^{7}$ W. Bossert (1989a): aOn the Extension of Preferences over a Set to the Power Set: an Axiomatic Characterization of a Quasi-orderings, Joumal of Economic Theoy, núm. 49, pp. 84-92.
K. Arrow, L. Hurwicz (1972): «An Optimality Cri. terion for Decision-making under Ignorances, en C. F. Carter y J. L. Ford (eds.), Uncertainy and Expectations in Economics: Essays in Honour of G.S.L. Shackle, Basil Blackwell, Oxford, 1-11.

' S. Barberà, C. Barrett y P. Pattanaik (1984): «On some Axioms for Ranking Sets of Alternativesm, Journal of Economic Theory, num. 33, pp. 301-308.

10 W. Bossert (1996): Uncertainy Aversion in Nonprobabilistic Decision Models, WP9609, Waterloo Economic Series.

" P. Pattanaik y B. Peleg (1984): «An Axiomatic Characterization of the Lexicographic Maximin Extension of an Ordering over a Set to the Power Setw, Social Choice and Welfare, num. 1, pp. 113-12.

"2 S. Nitzan y P. Pattanaik (1984): «Median-based Extension of an Ordering over a Set to the Power Set: an Axiomatic Characterizations, Soumal of Economic Theory, núm. 34, pp. 252-261.

is Vease, por ejemplo, A. K. Sen (1988): «Freedom of Choice: Concept and Content", Europ. Econ. Rev, num. 32, pp. 269-294; W. Bossert, P. K. Pattanaik e Y. Xu (1994); «Ranking Opportunity Sets: An Axiomatic Approach\%, Journal of Economic Theon, pp. 63-2, 326-345; M. Klemisch-Ahlert (1991): Freedom of Choice $A$ Comparison of Different Rankings of Opporturity Sets, DP 9104. Department of Economics, University of Osnabrück.

14 P. Pattanaik e Y. Xu (1990): «On Ranking Opportunity Sets in Terms of Freedom of Choice", Rech. Econ. Louvain núm. 56, pp. 383-390.

15 D. Kreps (1979): aA Representation Theorem for "Preference for Flexibility", Econometrica, núm. 47, pp. 565-577.

is Este resultado debe cualificarse si las preferencias sobre alternativas pueden ser tambiën preórdenes. Pero el espíritu del resultado se mantiene inalterado.

17 S. Barberà y B. Grodal, «Preference for Flexibility and the Opportunities of Choicem, mimeo. 